\title{
Large full band gaps for photonic crystals in two dimensions computed by an inverse method with multigrid acceleration
}

\author{
R. L. Chern, ${ }^{1,2}$ C. Chung Chang, ${ }^{2}$ Chien C. Chang, ${ }^{2}$ and R. R. Hwang ${ }^{1}$ \\ ${ }^{1}$ Institute of Physics, Academia Sinica, Taipei 115, Taiwan, Republic of China \\ ${ }^{2}$ Institute of Applied Mechanics, National Taiwan University, Taipei 106, Taiwan, Republic of China \\ (Received 21 April 2003; revised manuscript received 12 June 2003; published 29 August 2003)
}

\begin{abstract}
In this study, two fast and accurate methods of inverse iteration with multigrid acceleration are developed to compute band structures of photonic crystals of general shape. In particular, we report two-dimensional photonic crystals of silicon air with an optimal full band gap of gap-midgap ratio $\Delta \omega / \omega_{\text {mid }}=0.2421$, which is $30 \%$ larger than ever reported in the literature. The crystals consist of a hexagonal array of circular columns, each connected to its nearest neighbors by slender rectangular rods. A systematic study with respect to the geometric parameters of the photonic crystals was made possible with the present method in drawing a three-dimensional band-gap diagram with reasonable computing time.
\end{abstract}

DOI: 10.1103/PhysRevE.68.026704

PACS number(s): 02.70.Bf, 42.70.Qs, 78.20.Bh

\section{INTRODUCTION}

Photonic crystals with large full band gaps are of academic and practical interest [1,2]. Large full band gaps allow strong photon localization with the gap $[3,4]$, and a detailed manipulation of photonic defect states $[5,6]$. They have important applications such as defect cavities [7], optical waveguides [8], defect-mode photonic crystal lasers [9], and feedback mirror in laser diodes [10].

Because of the diversity of photonic crystals, there is a great demand for a general method for fast and accurate prediction of their band-gap structures. The most widely used method for this purpose is plane wave expansion [11-13], which has indeed provided useful information to band-gap structures. But there are several known disadvantages with this method: slow convergence $[14,15]$, the need to interpolate the dielectric property $[16,17]$, and sophisticated skills to diagonalize a dense matrix [18]. The purpose of this study is therefore twofold: one is to develop fast algorithms for computing band structures of photonic crystals, the other is to propose possible large full band-gap structures, the analysis of which requires major computational efforts.

Among various other possible techniques-transfer matrix method [19,20], multiple scattering method [21,22], finite difference time domain method [23,24], and finite element method [25,26] — finite difference multigrid method $[27,28]$ has the potential to meet the above standards. On the other hand, the studies $[29,30]$ provided an interesting algorithm toward optimization of band structures, but only in $E$ polarization or $H$ polarization. In the present study, a highly fast and accurate inverse method with multigrid acceleration is developed to be applicable to photonic crystals comprising of general-shape dielectric-dielectric materials. The operation count is a good order $N$ (the matrix size), where the proportional constant is dependent upon the shape and property of the materials used. Moreover, the developed algorithm is capable of resolving multieigenvalue band structures, and applicable to photonic crystals with interfaces of strong contrast. These salient features of the present method make it a highly efficient computational technique to analyze photonic crystals with two or more geometric parameters.

In this study, we further propose to consider a hexagonal lattice shown in Fig. 1. The reason of choosing this geometry is: previous studies indicate that band gaps for $E$ polarization are favored in a lattice of isolated high- $\varepsilon$ region, and band gaps for $H$ polarization are favored in a connected lattice [31]. A compromise must therefore be reached between the sizes of the dielectric column and the connecting rod in order to have a large full band gap for a given column radius. This is a problem with two geometrical parameters, i.e., the radius of the circular columns and the width of the connecting rods. Searching for their optimal values is often computationally demanding, but with the presently developed methods, this can be done with great efficiency. The rest of the paper provides a detailed description of the numerical algorithms and results of the computed band structures.

\section{BASIC EQUATIONS AND NUMERICAL METHODS}

For linear isotropic and frequency-independent dielectric materials with permeability close to one, the time-harmonic modes in two dimensions for $E$ polarization (TM) can be written as

$$
-\frac{1}{\varepsilon}\left(\frac{\partial^{2}}{\partial x^{2}}+\frac{\partial^{2}}{\partial y^{2}}\right) E=\left(\frac{\omega}{c}\right)^{2} E
$$

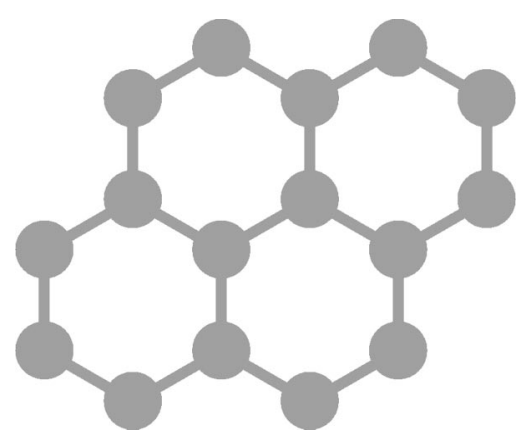

FIG. 1. Hexagonal array of circular columns and rods. 


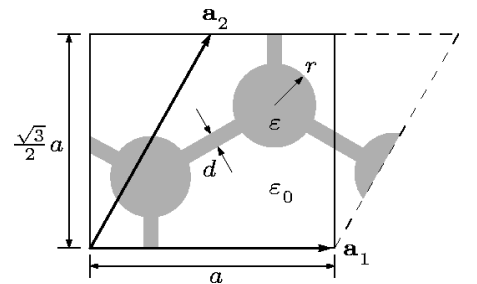

FIG. 2. Domain of computation for the hexagonal lattice.

and $H$ polarization (TE) as

$$
-\left[\frac{\partial}{\partial x}\left(\frac{1}{\varepsilon} \frac{\partial}{\partial x}\right)+\frac{\partial}{\partial y}\left(\frac{1}{\varepsilon} \frac{\partial}{\partial y}\right)\right] H=\left(\frac{\omega}{c}\right)^{2} H,
$$

where $E$ and $H$ are the electric and magnetic field intensities, respectively, and $\varepsilon=\varepsilon(\mathbf{r})$ is the dielectric function. To discretize Eqs. (1) and (2), a second-order central difference scheme is used. At the grid point near the interface, the dielectric function for $E$ polarization is interpolated as $[16,18]$

$$
\varepsilon=f_{a} \varepsilon_{a}+f_{b} \varepsilon_{b}
$$

and for $H$ polarization as

$$
\frac{1}{\varepsilon}=f_{a} \frac{1}{\varepsilon_{a}}+f_{b} \frac{1}{\varepsilon_{b}}
$$

where $\varepsilon_{a}$ and $\varepsilon_{b}$ are dielectric constants of materials $a$ and $b$, respectively, and $f_{a}$ and $f_{b}$ are fractions of the grid cell which contain $\varepsilon_{a}$ and $\varepsilon_{b}$, respectively. The domain of computation is chosen as a rectangle with the same area of one primitive cell of the hexagonal lattice as shown in Fig. 2. Bloch's theorem is applied at the domain boundary:

$$
\begin{gathered}
E_{\mathbf{k}}\left(\mathbf{r}+\mathbf{a}_{i}\right)=e^{i \mathbf{k} \cdot \mathbf{a}_{i}} E_{\mathbf{k}}(\mathbf{r}), \\
H_{\mathbf{k}}\left(\mathbf{r}+\mathbf{a}_{i}\right)=e^{i \mathbf{k} \cdot \mathbf{a}_{i}} H_{\mathbf{k}}(\mathbf{r}),
\end{gathered}
$$

where $E_{\mathbf{k}}$ and $H_{\mathbf{k}}$ are the Bloch functions for electric and magnetic fields, respectively, associated with the wave vector $\mathbf{k}$ in the first Brillouin zone, and $\mathbf{a}_{i}(i=1,2)$ is lattice translation vector.

From a practical point of view, the first few branches of eigenvalues are of primary interest. As a first step, it is natural for us to propose the method of inverse iteration to compute the eigenvalues as well as eigenvectors.

Let $\mathbf{A}$ be the discretization matrix of the differential operator in Eqs. (1) and (2). The basic idea is to solve (A $-\mu \mathbf{I}) \mathbf{x}=\mathbf{b}$ by inverse iteration $[32,33]$, where $\mu$ is chosen to be close to the eigenvalue one wishes to compute. The inverse algorithm is as follows:

InverseEigen \{

for $n=1$ to $S$

Initial guess $\mathbf{b}$

do

Solve $(\mathbf{A}-\mu \mathbf{I}) \mathbf{x}=\mathbf{b}$ by $\mathbf{L U D}$ or PCG

Deflate $\mathbf{x}$ by $\mathbf{q}_{1}$ to $\mathbf{q}_{n-1}$

Set $\mathbf{b}=\mathbf{x} /\|\mathbf{x}\|$

Rayleigh Quotient $\lambda_{n}=\langle\mathbf{b}, \mathbf{A} \mathbf{b}\rangle$
TABLE I. Computing times in CPU seconds for six bands.

\begin{tabular}{lcc}
\hline \hline$N_{\text {grid }} / N_{\text {wave }}$ & FMGI & PWE \\
\hline $32 \times 32$ & 0.03 & 5.80 \\
$48 \times 48$ & & 57.06 \\
$64 \times 64$ & 0.17 & 339.53 \\
$128 \times 128$ & 0.74 & $12836($ est. $)$ \\
$256 \times 256$ & 2.74 & \\
$512 \times 512$ & 9.39 & \\
$1024 \times 1024$ & 33.59 & \\
\hline \hline
\end{tabular}

end $\}$

$$
\begin{aligned}
& \text { until }\left\|\left(\mathbf{A}-\lambda_{n} \mathbf{I}\right) \mathbf{b}\right\|_{2}<\boldsymbol{\epsilon} \\
& \text { Set } \mathbf{q}_{n}=\mathbf{b}
\end{aligned}
$$

where $\lambda_{1} \leqslant \lambda_{2} \leqslant \ldots \leqslant \lambda_{S}$ is the sequence of smallest eigenvalues, and $\mathbf{q}_{1}, \ldots, \mathbf{q}_{S}$ are the corresponding eigenvectors. The inner product $\langle\cdot, \cdot\rangle$ is defined as

$$
\langle\mathbf{x}, \mathbf{y}\rangle=\sum_{i} w_{i} \bar{x}_{i} y_{i}, w_{i}= \begin{cases}\varepsilon\left(\mathbf{r}_{i}\right) & \text { for } E \text { polarization } \\ 1 & \text { for } H \text { polarization }\end{cases}
$$

in view of the different orthogonal properties of $E$ field and $H$ field:

$$
\begin{gathered}
\frac{1}{V} \int_{V} E_{m}^{*}(\mathbf{r}) \varepsilon(\mathbf{r}) E_{n}(\mathbf{r}) d \mathbf{r}=\delta_{m n}, \\
\frac{1}{V} \int_{V} H_{m}^{*}(\mathbf{r}) H_{n}(\mathbf{r}) d \mathbf{r}=\delta_{m n},
\end{gathered}
$$

where $E_{n}$ and $H_{n}$ are the $n$th eigenmodes of the electric and magnetic fields, respectively. $V$ denotes the volume of the primitive cell and $\delta_{m n}$ is the Kronecker delta. LUD denotes the lower-upper decomposition solver for matrix inversion and PCG the preconditioned conjugate gradient method.

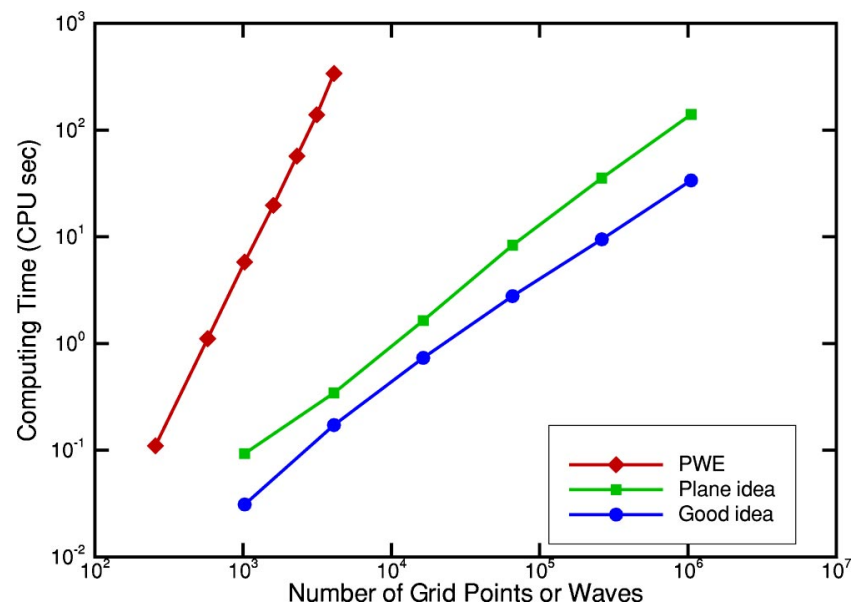

FIG. 3. Comparison of PWE, a plain idea and a good idea. The plain idea embeds the multigrid solver in the inverse iteration loop, while the good idea embeds the inverse iteration in the multigrid acceleration structure. 


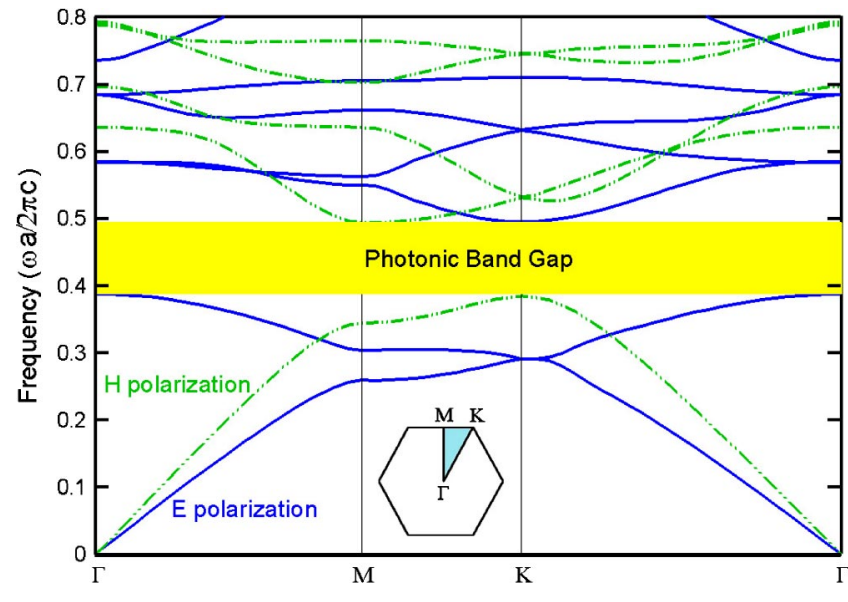

FIG. 4. Band structures by $256 \times 256$ grid for the hexagonal lattice in Fig. $1\left(\varepsilon / \varepsilon_{0}=13, r / a=0.155\right.$, and $\left.d / a=0.035\right)$.

Next, we propose two ideas to accelerate the convergence of matrix inversion in the algorithm. A plain idea is to embed a multigrid solver in the inverse iteration loop, namely, multigrid takes place of the expensive LUD. A good idea is to embed inverse iteration in the multigrid acceleration structure. In this idea, the Rayleigh quotient is updated at each grid level, which results in a more efficient algorithm. The basic idea of multigrid method $[34,35]$ is to solve the matrix problem by approximating the solution on a fine grid, solving the residue on a coarse grid, and then improving the solution on the fine grid. Successively applying this idea on each level of grids causes relaxation of errors on different resolution, and hence accelerates the convergence.

Let there be $L$ levels of grids. On each grid level $m$, the differential operator is discretized to form the matrix $\mathbf{A}^{(m)}$. The multilevel algorithm based on the good idea is as follows:

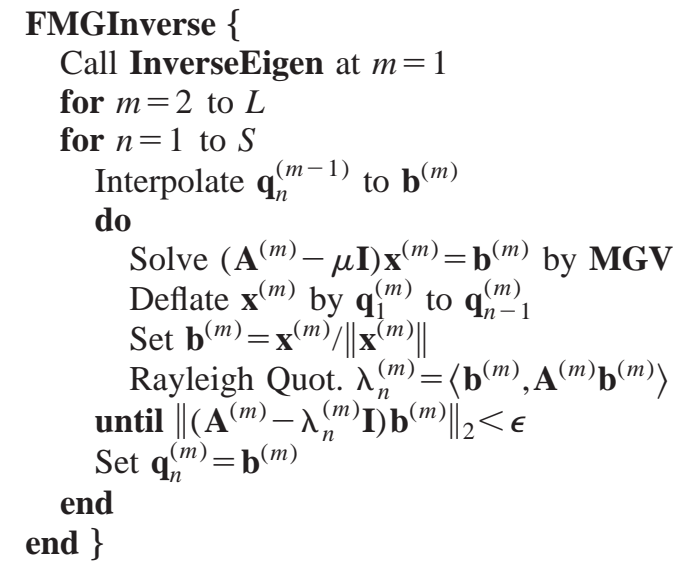

TABLE II. Convergence test for maximum band gap against the grid size.

\begin{tabular}{lccccc}
\hline \hline$N_{\text {grid }}$ & $64^{2}$ & $128^{2}$ & $256^{2}$ & $512^{2}$ & $1024^{2}$ \\
\hline$a \omega_{\text {up }} / 2 \pi c$ & 0.4917 & 0.4940 & 0.4940 & 0.4941 & 0.4940 \\
$a \omega_{\text {low }} / 2 \pi c$ & 0.3946 & 0.3876 & 0.3873 & 0.3873 & 0.3873 \\
$a \omega_{\text {mid }} / 2 \pi c$ & 0.4431 & 0.4408 & 0.4407 & 0.4407 & 0.4407 \\
$a \Delta \omega / 2 \pi c$ & 0.0971 & 0.1064 & 0.1067 & 0.1068 & 0.1067 \\
$\Delta \omega / \omega_{\text {mid }}$ & 0.2191 & 0.2413 & 0.2421 & 0.2423 & 0.2421 \\
\hline \hline
\end{tabular}

TABLE III. Comparison of the gap-midgap ratios for twodimensional photonic crystals.

\begin{tabular}{lcccc}
\hline \hline & $\varepsilon / \varepsilon_{0}$ & $a \omega_{\text {mid }} / 2 \pi c$ & $a \Delta \omega / 2 \pi c$ & $\Delta \omega / \omega_{\text {mid }}$ \\
\hline Present & 13 & 0.4407 & 0.1067 & 0.2421 \\
& 11.4 & 0.4503 & 0.0888 & 0.1972 \\
{$[31]$} & 13 & & & 0.186 \\
{$[36]$} & 11.4 & $(0.71)$ & 0.1158 & $(0.1631)$ \\
{$[37]$} & 12.25 & & 0.0872 & 0.182 \\
{$[38]$} & 11.4 & $(\sim 0.45)$ & 0.0762 & $(\sim 0.1693)$ \\
{$[39]$} & 12.96 & & 0.090 & 0.171 \\
{$[40]$} & 11.4 & $>0.9$ & 0.0967 & $<0.1074$ \\
& & $>0.8$ & 0.0849 & $<0.1061$ \\
\hline \hline
\end{tabular}

where MGV denotes the well-known multigrid $V$-cycle solver in numerical linear algebra [34,35].

The whole idea of proposing an inverse method with multigrid acceleration for computing photonic band structures has now become clear. First of all, the interface interpolations (3) and (4) are used to take care of strong dielectric contrasts. Second, the deflation in the algorithm of inverse iteration enables singling out the eigenvalues one by one from the smallest even when they may be degenerated. Third, interlacing the inverse iteration and multigrid acceleration makes the whole method an amazingly fast algorithm for computing photonic band structures, as demonstrated below.

\section{RESULTS AND DISCUSSION}

As an example of test, we compute the first six frequency bands at the point $X$ of a square array of dielectric columns with radius $r / a=0.45$ and dielectric contrast $\varepsilon / \varepsilon_{0}=13$. Table I shows a comparison of computing times in central processing unit (CPU) seconds between the present method (FMGI) and plain plane wave expansion (PWE). The comparison is made by averaging the computing times over 68 wave numbers. Both computations are performed on a PC

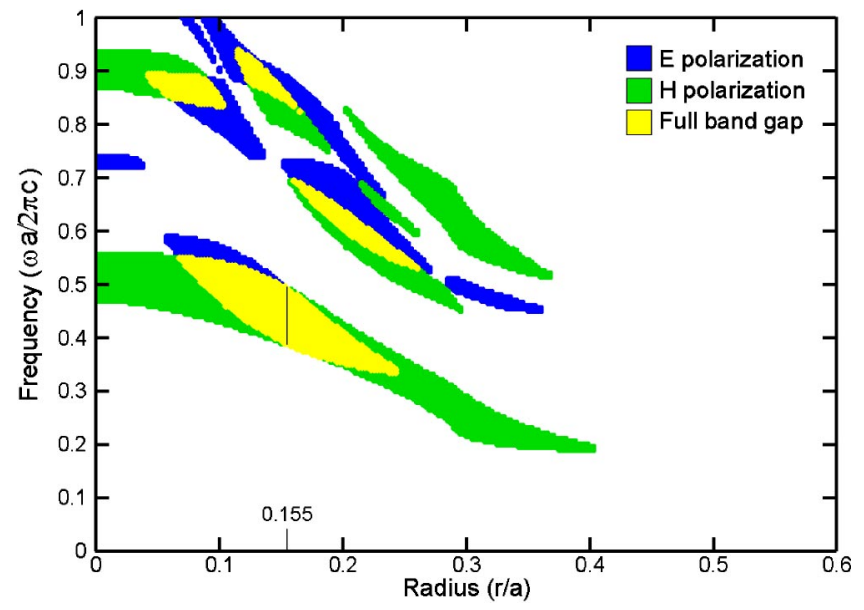

FIG. 5. A map of band gaps by $128 \times 128$ grid for the hexagonal lattice in Fig. 1 by varying radius $r / a$ of circular columns. The dielectric contrast $\varepsilon / \varepsilon_{0}$ is 13 with fixed $d / a=0.035$. 


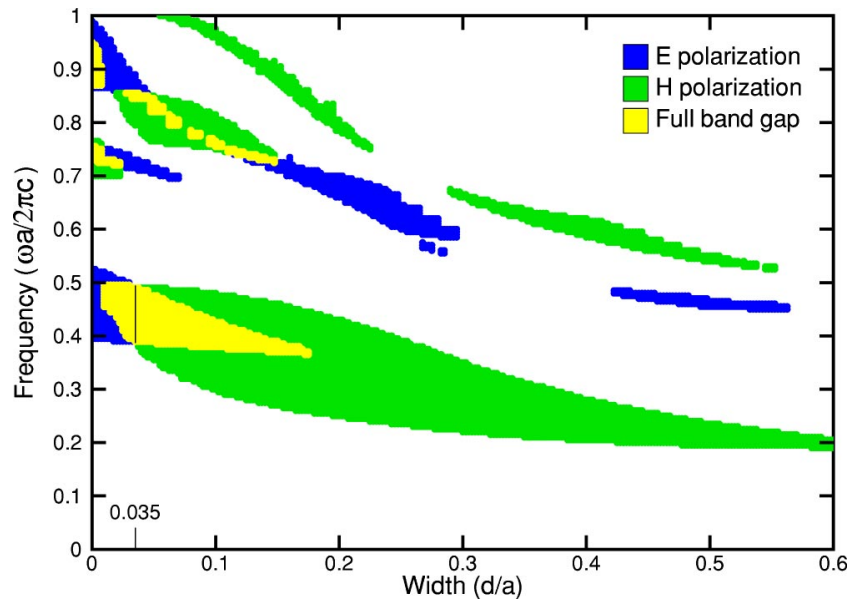

FIG. 6. A map of band gaps by $128 \times 128$ grid for the hexagonal lattice in Fig. 1 by varying width $d / a$ of connecting rods. The dielectric contrast $\varepsilon / \varepsilon_{0}$ is 13 with fixed $r / a=0.155$.

Pentium 4. The computing time for the present method is of a good order $N=N_{\text {grid }}$, and tends to be superlinear at large $N$. The plane wave expansion, on the contrary, has the computational cost of about the order $N^{2.873}$. Figure 3 shows a comparison of PWE, the plain idea and the good idea of implementing inverse iteration with multigrid acceleration. The good-idea algorithm is roughly four times faster than the plain-idea algorithm, and even better at larger $N$. Nevertheless, both of them are faster than PWE by almost two orders in $N_{\text {grid }}$.

The fast algorithm developed above enables efficient search for the optimal geometry of the photonic crystals in Fig. 1. The largest gap-midgap ratio for silicon air $\left(\varepsilon / \varepsilon_{0}\right.$ $=13$ ) corresponds to $r / a=0.155$ and $d / a=0.035$. Figure 4 shows the detailed band structure at these ratios. It is interesting to notice that the full band gap is almost the simultaneous maximum band gap of both $E$ and $H$ polarizations. In order to ensure the accuracy of the band gap, computations are performed on five different grids. Table II lists the nu-

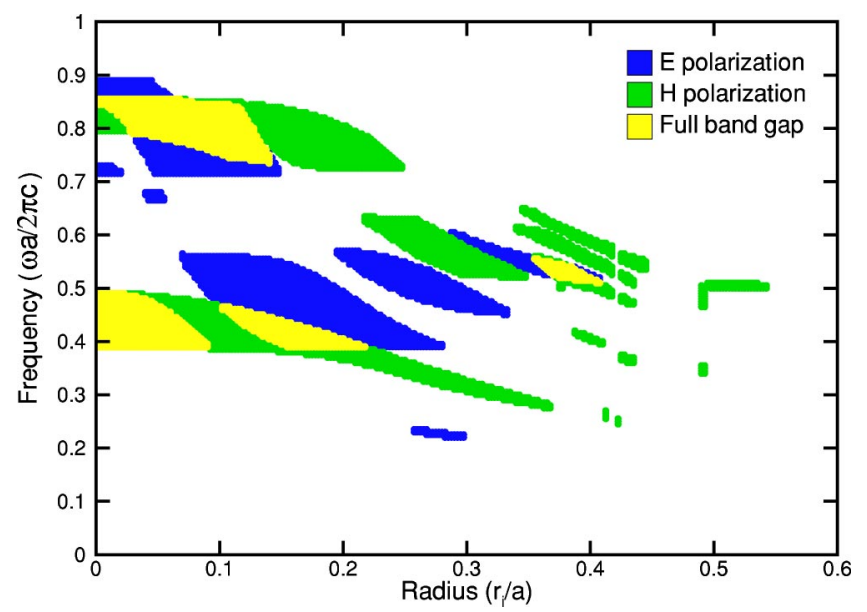

FIG. 7. A map of band gaps by $128 \times 128$ grid for the hexagonal lattice in Fig. 1 by varying radius $r_{i} / a$ of the inserted center columns. The dielectric contrast $\varepsilon / \varepsilon_{0}$ is 13 with fixed $r / a=0.155$ and $d / a=0.035$.

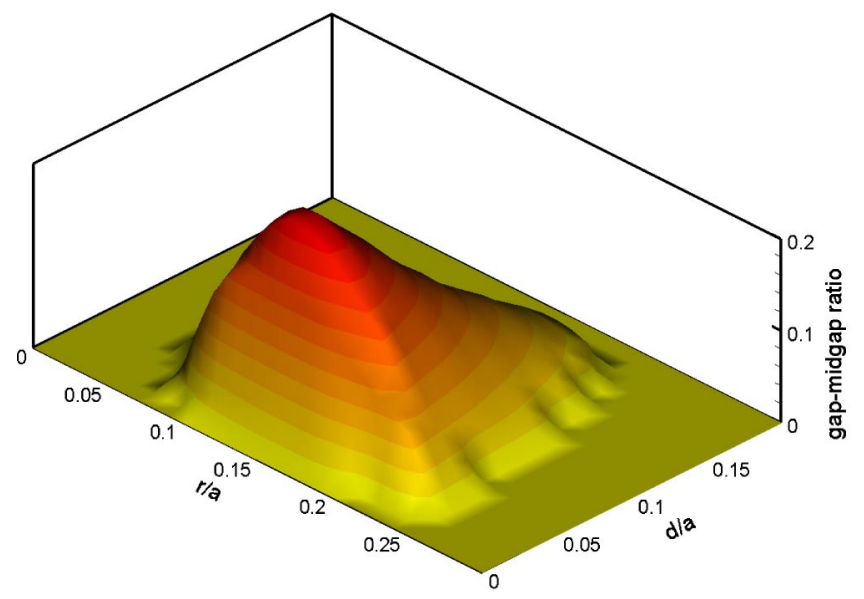

FIG. 8. A three-dimensional terrain of the major full band gaps for $0 \leqslant r / a \leqslant 0.3$ and $0 \leqslant d / a \leqslant 0.2$.

merics of the location of the maximum full band gap for different grids for $N \geqslant 128 \times 128$. In this paper, most of the computations are performed with the grid $N=128 \times 128$. All the computed results coincide with each other to three significant digits. Table III has a list of references that reported numerics of large band gaps. It is found that for GaAs air $\left(\varepsilon / \varepsilon_{0}=11.4\right)$ the present $\Delta \omega / \omega_{\text {mid }}=0.1972$ is the largest, while for silicon air the present $\Delta \omega / \omega_{\text {mid }}=0.2421$ is not only the largest but 30\% larger than reported in Ref. [31].

Figure 5 is a gap map versus $r / a$ (with fixed $d / a$ $=0.035$ ). This map is obtained by computing the band structures at every 0.0025 of $r / a$. Figure 6 is a gap map versus $d / a$ (with fixed $r / a=0.155$ ). This gap map is obtained by computing the band structures at every 0.0025 of $d / a$. The two maps show clearly the location of the full band gap with maximum gap-midgap ratio. In particular, by increasing $d / a$ from 0.035 , the full band gap is gradually embedded into a large band gap of $H$ polarization. By varying $r / a$ near 0.155 , the band gaps of $E$ polarization and of $H$ polarization have a good overlap with each other. It is noted that addition of a smaller column in the center of the unit cell of a square/ honeycomb lattice for symmetry reduction can increase the full band gap [40]. However, the present study does not find further increase of the full band gap by inserting a circular column, no matter what its radius is, in the center of the photonic structure of Fig. 1. Figure 7 shows the gap map. As $r_{i} / a$ ( $r_{i}$ : the radius of the inserted column) increase from 0 , the full band gap shrinks gradually in size due to the decrease of the band gap in $E$ polarization. Figure 8 is the result of all elaboration plotted in a three-dimensional band-gap diagram. It gives an overall outlook of the major full band gap, and shows the most sensitive direction to geometric parameters. This plot is composed of as many band gaps as computed for 61 values of $r / a$ and 41 values of $d / a$, each for $E$ and $H$ polarizations. There are totally 60024 eigenvalues computed.

\section{CONCLUDING REMARKS}

In conclusion, we have developed two fast inverse methods with multigrid acceleration for computing photonic band 
structures in two dimensions. The fast algorithms are capable of resolving multieigenvalue band structures, and applicable to photonic crystals with interfaces of strong contrast. In particular, the good idea algorithm is even faster than the plane idea algorithm. The methods enable us to explore photonic band structures with two or more geometric parameters systematically. In addition, we propose a photonic structure that has significantly larger full band gaps compared to those ever reported in the literature. The study has illustrated that optimal design of photonic structures can benefit greatly from physical principle, aided by fast computation. Presently, the methods are applied to two-dimensional photonic crystals. In principle, they can be extended to problems in three dimensions. Three-dimensional problems are, however, more challenging by presenting themselves as eigenproblems of vector operators. One such extension is currently being under intensive investigation, and the results will be reported elsewhere.

\section{ACKNOWLEDGMENT}

This work was supported in part by the National Science Council of the Republic of China under Contract No. NSC 90-2212-E-002-238.
[1] E. Yablonovitch, Phys. Rev. Lett. 58, 2059 (1987).

[2] S. John, Phys. Rev. Lett. 58, 2486 (1987).

[3] N. Garcia and A.Z. Genack, Phys. Rev. Lett. 66, 1850 (1991).

[4] S. John, Phys. Today 44(5), 32 (1991).

[5] S. Lin and G. Arjavalingam, J. Opt. Soc. Am. B 11, 2124 (1994).

[6] T. Krauss et al., Nature (London) 383, 699 (1996).

[7] J.S. Foresi, P.R. Villeneuve, J. Ferrera, E.R. Thoen, G. Steinmeyer, S. Fan, J.D. Joannopoulos, L.C. Kimerling, H.I. Smith, and E.P. Ippen, Nature (London) 390, 143 (1997).

[8] A. Mekis, J.C. Chen, I. Kurland, S. Fan, P.R. Villeneuve, and J.D. Joannopoulos, Phys. Rev. Lett. 77, 3787 (1996).

[9] O. Painter, R.K. Lee, A. Scherer, A. Yariv, J.D. OBrien, P.D. Dapkus, and I. Kim, Science 284, 1819 (1999).

[10] D.L. Bullock, C. Shih, and R.S. Margulies, J. Opt. Soc. Am. B 10, 399 (1993).

[11] K.M. Leung and Y.F. Liu, Phys. Rev. Lett. 65, 2646 (1990).

[12] Z. Zhang and S. Satpathy, Phys. Rev. Lett. 65, 2650 (1990).

[13] K.M. Ho, C.T. Chan, and C.M. Soukoulis, Phys. Rev. Lett. 65, 3152 (1990).

[14] H.S. Sözüer, J.W. Haus, and R. Inguva, Phys. Rev. B 45, 13 962 (1992).

[15] L. Shen and A. He, J. Opt. Soc. Am. A 19, 1021 (2002).

[16] R.D. Meade, A.M. Rappe, K.D. Brommer, J.D. Joannopoulos, and O.L. Alherhand, Phys. Rev. B 48, 8434 (1993).

[17] P. Lalanne, Phys. Rev. B 58, 9801 (1998).

[18] S.G. Johnson and J.D. Joannopoulos, Photonic Crystals (Kluwer Academic, Boston, 2002).

[19] J.B. Pendry and A. MacKinnon, Phys. Rev. Lett. 69, 2772 (1992).

[20] M.M. Sigalas, C.T. Chan, K.M. Ho, and C.M. Soukoulis, Phys. Rev. B 52, 11744 (1995).
[21] X. Wang, X.-G. Zhang, Q. Yu, and B.N. Harmon, Phys. Rev. B 47, 4161 (1993).

[22] K.M. Leung and Y. Qiu, Phys. Rev. B 48, 7767 (1993).

[23] C.T. Chan, Q.L. Yu, and K.M. Ho, Phys. Rev. B 51, 16635 (1995).

[24] A.J. Ward and J.B. Pendry, Phys. Rev. B 58, 7252 (1998).

[25] D.C. Dobson, J. Comput. Phys. 149, 363 (1999).

[26] W. Axmann and P. Kuchment, J. Comput. Phys. 150, 468 (1999).

[27] D. Hermann, M. Frank, K. Busch, and P. Wolfle, Opt. Express 8, 167 (2001).

[28] A. Brandt, S. McCormick, and J. Ruge, SIAM (Soc. Ind. Appl. Math.) J. Sci. Stat. Comput. 4, 244 (1983).

[29] S.J. Cox and D.C. Dobson, SIAM (Soc. Ind. Appl. Math.) J. Appl. Math. 59, 2108 (1999).

[30] S.J. Cox and D.C. Dobson, J. Comput. Phys. 158, 214 (2000).

[31] J.D. Joannoupoulos, R.D. Meade, and J.N. Winn, Photonic Crystals (Princeton University Press, Princeton, 1995).

[32] B.N. Parlett, The Symmetric Eigenvalue Problem (SIAM, Philadelphia, 1998).

[33] G.H. Golub and C.F. Van Loan, Matrix Computations, 3rd ed. (Johns Hopkins University Press, London, 1996).

[34] J.W. Demmel, Applied Numerical Linear Algebra (SIAM, Philadelphia, 1997).

[35] U. Trottenberg, C. Oosterlee, and A. Schüller, Multigrid (Academic Press, London, 2001).

[36] L. Shen, S. He, and S. Xiao, Phys. Rev. B 66, 165315 (2002).

[37] N. Susa, J. Appl. Phys. 91, 3501 (2002).

[38] M. Qiu and S. He, J. Opt. Soc. Am. B 17, 1027 (2000).

[39] X.H. Wang, B.Y. Gu, Z.Y. Li, and G.Z. Yang, Phys. Rev. B 60, 11417 (1999).

[40] C.M. Anderson and K.P. Giapis, Phys. Rev. Lett. 77, 2949 (1996). 